\title{
Galenus 2012
}

\section{Die Jury hat entschieden}

\author{
Für manche ist er der inoffizielle "Nobelpreis" für Pharmakologie: der \\ Galenus-von-Pergamon-Preis. In Berlin wurde er kürzlich erneut \\ verliehen - an drei beeindruckende Preisträger.
}

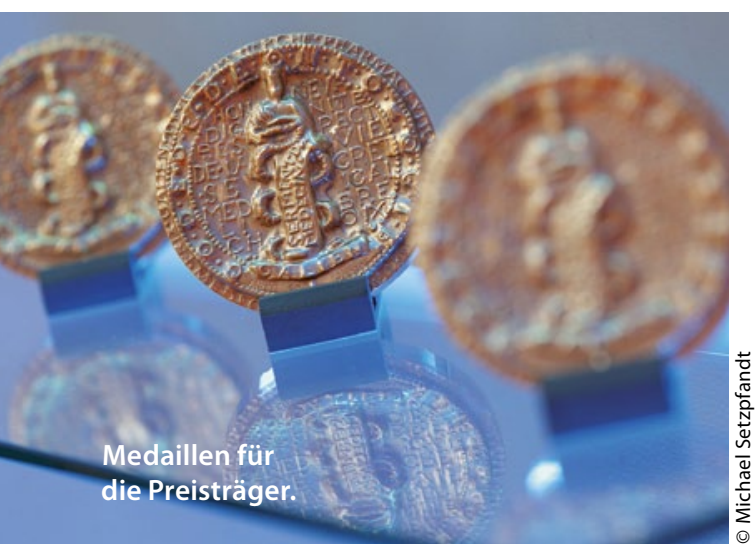

A m 18. Oktober war es wieder so weit: Im Rahmen einer feierlichen Gala wurde der begehrte Galenus-von-Pergamon-Preis verliehen. Drei Preise für exzellente Grundlagenforschung in der Pharmakologie und herausragende pharmazeutische Innovationen.

Ausgezeichnet wurden Gilenya ${ }^{\oplus}$ (Fingolimod) von Novartis Pharma in der Kategorie Primary Care und Zelboraf (Vemurafenib) von Roche Pharma in der Kategorie Specialist Care. In der Kategorie Grundlagenforschung wurde das Team um Dr. Thomas Worzfeld aus Bad Nauheim für die Entwicklung eines neuen Ansatzes zur Therapie bei metastasierendem Brustkrebs geehrt.

Die 14-köpfige Jury unter dem Vorsitz von Prof. Erland Erdmann, Köln, tat sich bei ihrer Entscheidung schwer, wie der Jury-Präsident sagte. „Alle diesjährigen Kandidaten sind Innovationen." Mit Mehrheit hätten sich die Juroren schließlich für die diesjährigen Galenus-Gewinner entschieden.

\section{Innovationen erreichen den Patienten schnell}

Fingolimod ist ein orales Medikament zur Therapie von Patienten mit Multipler
Sklerose (MS). Es ist zugelassen für bisher nicht behandelte Patienten, die an einer rasch fortschreitenden, schwer schubförmigen MS erkrankt sind, sowie zur Eskalationstherapie, wenn trotz Behandlung mit einem Beta-Interferon eine hohe Krankheitsaktivität vorliegt. Mit Fingolimod gelingt es, die Schubrate zu verringern. Im Vergleich zur Behandlung mit Interferon ließ sich die Schubrate in einer Zulassungsstudie um 52\% senken (0,33 vs. 0,16 Schübe pro Jahr).

Vemurafenib ist die erste Option für eine personalisierte Therapie bei inoperablem oder metastasiertem Melanom. Das Medikament in Form von Filmtabletten ist zugelassen zur Therapie von Melanompatienten, deren Tumoren ein mutiertes BRAF-Gen aufweisen.

In der Zulassungsstudie betrug die geschätzte mediane progressionsfreie Überlebenszeit 5,3 Monate. Im Vergleich: Bei Patienten, die Dacarbazin als Standardtherapie erhalten hatten, betrug sie nur 1,6 Monate. In der Vemurafenib-Gruppe war zudem das Sterberisiko um 63 Prozent und das Progressionsrisiko um 74 Prozent verringert. Die erste Phase der Entwicklung des Präparates erfolgte durch das US-Unternehmen Plexxikon.

\section{Grundlagenforschung zu \\ Brustkrebs}

Der Galenus-Preisträger Worzfeld und sein Team fanden heraus, dass der Rezeptor Plexin-B1 eine besondere Bedeutung für die Metastasierung bei Brustkrebs hat. Mammakarzinom-Patientinnen haben eine umso bessere Überlebenschance, je weniger Plexin-B1 exprimiert wird. Inzwischen wird ein monoklonaler Antikörper gegen diesen Rezeptor präklinisch getestet wird.

„Anfangs dachten wir nicht, dass wir mit unserer Forschung bei Brustkrebs
CharityAward für

Kinderhospiz Balthasar

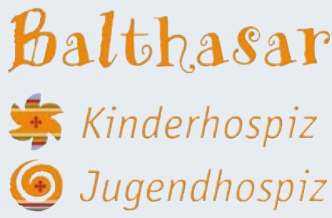

Auch der Springer Medizin CharityAward 2012 wurde bei der Gala verliehen. Ausgezeichnet wurde das Balthasar Kinder- und Jugendhospiz für sein unermüdliches Engagement. Träger des Hospizes ist die Gemeinnützige Gesellschaft der Franziskanerinnen zu Olpe. Schirmherr und Bundesgesundheitsminister Daniel Bahr überreichte den mit 150.000 Euro dotierten Preis.

1998 gegründet, ist Balthasar das erste Kinder- und Jugendhospiz in Deutschland. Die Unterhaltung einer solchen Einrichtung birgt immer wirtschaftliche Risiken, daher freut sich Hospizleiter Rüdiger Barth: „Wir sind stolz und glücklich über den Springer Medizin CharityAward für das Kinder- und Jugendhospiz Balthasar und klartext! Durch das Medienpaket können wir unmittelbar Spenden generieren und zugleich auch neue Kontakte knüpfen, die vielleicht in eine langfristige Unterstützung münden. Eine doppelte Hilfe, für die wir all jenen danken möchten, die für uns gestimmt haben. Maurice aus dem Kinderhospiz beschreibt Balthasar so: ,Ich muss nicht lange nach einem besonderen Augenblick suchen. Ich kann ihn jetzt und hier finden.' Danke, dass Sie dazu beitragen."

Der Springer Medizin CharityAward umfasst einen Barscheck über 50.000 Euro und ein Medienpaket über weitere 100.000 Euro. Das Medienpaket bedeutet individuelle Dienstleistung für den Gewinner: Die Fachverlagsgruppe unterstützt den Gewinner gezielt bei seiner zukünftigen Öffentlichkeitsarbeit. Peter Leiner

landen würden“, so Worzfeld, der die Auszeichnung aus den Händen der Galenus-Vizepräsidentin Prof. Marion Kiechle, München, entgegennahm. Ausgangspunkt seien neurowissenschaftliche Fragestellungen gewesen. Geplant sind Untersuchungen mit dem Antikörper auch beim Magen- und beim Ovarialkarzinom.

Peter Leiner 